\title{
Toxicity of daily low dose cisplatin in radiochemotherapy for locally advanced head and neck cancer
}

\author{
Hendrik Andreas Wolff · Tobias Overbeck • Ralph M. Roedel • Robert M. Hermann • \\ Markus K. A. Herrmann · Tereza Kertesz • Hilke Vorwerk • Andrea Hille • \\ Christoph Matthias $\cdot$ Clemens F. Hess $\cdot$ Hans Christiansen
}

Received: 14 October 2008 / Accepted: 4 December 2008 / Published online: 24 December 2008

(C) The Author(s) 2008. This article is published with open access at Springerlink.com

\begin{abstract}
Purpose To evaluate toxicity of radiochemotherapy schedule using daily-low-dose-cisplatin in radiochemotherapy of locally-advanced head-and-neck-cancer (HNSCC). Methods and patients From October 2003 to October 2006, 50 patients with HNSCC (stage III/IVA/IVB) were treated. In 32 patients, surgery and adjuvant radiotherapy (64 Gy), in 18 patients definitive radiotherapy $(70 \mathrm{~Gy})$ was performed. Low-dose-cisplatin was applied concomitantly ( $6 \mathrm{mg} / \mathrm{m}^{2} /$ every radiotherapy-day).

Results Acute toxicity $\geq$ grade 3 was observed in 22 patients (11 patients mucositis/dysphagia, 7 hematologic toxicity, 4 mucositis/dysphagia/hematologic toxicity). $90 \%$ of our patients received $>80 \%$ of the planned cumulative chemotherapy dose, $94 \%$ the intended dose of radiotherapy. After median follow-up of 24.2 months, 3-year overall survival and loco-regional control rates were 67.1 and $78 \%$. During follow-up, chronic toxicity $\geq$ grade 3 (xerostomia, subcutaneous fibrosis, or lymphedema) was observed in nine patients.

Conclusion We found chemoradiation with daily-lowdose-cisplatin to be feasible with advantage of low acute
\end{abstract}

H. A. Wolff $(\bowtie) \cdot$ R. M. Hermann · M. K. A. Herrmann .

T. Kertesz $\cdot$ H. Vorwerk - A. Hille $\cdot$ C. F. Hess $\cdot$ H. Christiansen

Department of Radiotherapy and Radiooncology,

Universitätsmedizin Göttingen, Robert-Koch-Strasse 40,

37075 Göttingen, Germany

e-mail: drhawolff@googlemail.com

T. Overbeck

Department of Hematology and Oncology,

Universitätsmedizin Göttingen, Göttingen, Germany

R. M. Roedel · C. Matthias

Department of Otolaryngology,

Universitätsmedizin Göttingen, Göttingen, Germany and chronic toxicity. Therefore, use of low-dose-cisplatin should be evaluated in future clinical trials.

Keywords Low dose cisplatin $\cdot$ HNSCC . Radiochemotherapy $\cdot$ Toxicity

\section{Introduction}

Radiotherapy is an important therapeutic modality in the treatment of advanced (UICC stage III and IVA/IVB) head neck squamous cell carcinoma (HNSCC) in curative intent and is used either as adjuvant or primary treatment modality. It has been proven that concomitant chemotherapy improves overall survival as well as loco-regional control both in the primary and in the adjuvant situation (Bauchaud et al. 1996; Bernier et al. 2004; Browman et al. 2001; Cooper et al. 2004; El-Sayed and Nelson 1996; Pignon et al. 2000). However, concomitant chemotherapy also increases the risk of escalated acute toxicity. The optimal chemotherapy regimen has not been evaluated, yet. Common regimens besides several others are cisplatin $100 \mathrm{mg} / \mathrm{m}^{2}$ on days 1,22 , and 43 of radiotherapy (Rades et al. 2008); $40 \mathrm{mg} / \mathrm{m}^{2}$ weekly during radiotherapy (Geeta et al. 2006), or $20 \mathrm{mg} / \mathrm{m}^{2}$ for 4 days in week 1 and 5 of radiotherapy (Lau et al. 2006), optionally combined with 5-FU (Adelstein et al. 2006). A relatively infrequent used regimen in radiochemotherapy for head and neck cancer is the use of daily low dose cisplatin, which includes the administration of cisplatin $6 \mathrm{mg} / \mathrm{m}^{2}$ on each radiotherapy day. However, this application form is well established in the treatment of locally advanced non small cell lung cancers with primary radiochemotherapy (Pradier et al. 2005; Schaake-Koning et al. 1992; Semrau et al. 2007; Takiguchi et al. 2005). The experiences obtained from these studies regarding the treatment of NSCLC show that good 
treatment outcome with acceptable toxicity can be achieved with low dose cisplatin compared to radiotherapy alone. So far, there are only few reports on efficacy and toxicity of low dose cisplatin in radiochemotherapy for advanced HNSCC (Hoebers et al. 2007; Jeremic et al. 2004; Jeremic and Milicic 2008). Therefore, the purpose of this study was to evaluate the toxicity in patients treated with this regimen for locally advanced HNSCC either in the primary or adjuvant situation in our department.

\section{Methods and patients}

From October 2003 to October 2006, all stage III/IVA/B HNSCC patients treated in our department (primary or adjuvant) were designated to receive concomitant cisplatin $6 \mathrm{mg} / \mathrm{m}^{2}$ on each radiotherapy day. No other cisplatin regimens were used in our department during the respective time period as patients, who were not able to receive cisplatin due to a reduced creatinine clearance $(\leq 60 \mathrm{ml} / \mathrm{min})$, which was determined in every case before starting treatment, were either selected to receive radiotherapy alone or radiotherapy plus cetuximab in the primary situation. In summary, 50 patients with locally advanced HNSCC received concomitant radiochemotherapy with cisplatin $6 \mathrm{mg} / \mathrm{m}^{2}$ on each radiotherapy day in the respective time period and therewith qualified for inclusion in the presented study. All tumors were histologically determined as squamous cell carcinoma (41 histologic grade 2 and 9 grade 3 ). Forty-eight patients were males and two females, patients age ranged from 43 to 80 years (median 61 years). Tumors were localized as follows: oral cavity (6), oropharynx (29), hypopharynx (7), and larynx (8). Disease was staged according to the Union Internationale Contre le Cancer/ American Joint Committee on Cancer (UICC/AJCC) criteria. Eight patients were in stage III, 35 patients were in stage IVA, and 7 patients were in stage IVB. Thereby, in 2 patients the primary tumor was staged as $\mathrm{T} 1$, in 7 patients as $\mathrm{T} 2$, in 15 patients as $\mathrm{T} 3$, and in 26 patients as T4. In summary, 43 patients presented with histologically proven positive cervical lymph nodes ( 6 patients $\mathrm{N} 1,30$ patients $\mathrm{N} 2$, and 7 patients N3). One day before the start of radiochemotherapy, the hemoglobin level was determined in each patient, and its median was $8.9 \mathrm{mmol} / \mathrm{l}(13.9 \mathrm{~g} / \mathrm{dl})$ [range 6-11.5 mmol/l $(9.3-17.9 \mathrm{~g} / \mathrm{dl})]$. Four male patients presented with an anemia grade 1 according to the CTC score before radiochemotherapy. These patients received blood preservations to correct anemia before starting treatment. Pretreatment characteristics of patients entered in study are concluded in Table 1.

Initial examinations before treatment included medical history, clinical ENT (ear-nose-throat) examination (magnifying laryngoscopy, upper bronchoscopy, esophagoscopy,
Table 1 Pretreatment characteristics of patients entered in study

\begin{tabular}{ll}
\hline Characteristic & No. patients $(\%)$ \\
\hline Gender & \\
Male & $48(96)$ \\
Female & $2(4)$ \\
Tumor localization & \\
Oral cavity & $6(12)$ \\
Oropharynx & $29(58)$ \\
Hypopharynx & $7(14)$ \\
Larynx & $8(16)$ \\
Stage & \\
III & $8(16)$ \\
IVA & $35(70)$ \\
IVB & $7(14)$ \\
Histologic grade & \\
1 & $0(0)$ \\
2 & $41(82)$ \\
3 & $9(18)$ \\
Hemoglobin level before treatment & \\
$>8.32$ mmol/l $(>13.9 \mathrm{~g} / \mathrm{dl})$ & $23(54)$ \\
$<8.32$ mmol/l $(<13.9 \mathrm{~g} / \mathrm{dl})$ & $27(46)$ \\
Surgery & \\
Yes & $38(76)$ \\
No & $12(24)$ \\
\hline
\end{tabular}

ear-nose-throat endoscopy) with biopsies in potential mucosal primary sites, complete blood counts, biochemical analysis including creatinine clearance, electrocardiogram, chest X-rays, abdominal ultrasound, and CT scans of the thorax and the head and neck with contrast medium.

A total of 32 patients underwent curative surgery as a primary treatment followed by adjuvant radiochemotherapy up to a total dose of $64 \mathrm{~Gy}$. Twenty-five of these patients had histologically proven involved lymph nodes; extracapsular extension of lymph nodes was detected in six of these patients. Eighteen inoperable patients were treated with primary radiochemotherapy to a total dose of $70 \mathrm{~Gy}$. Thereby, all patients received conventional fractionated 3-D conformal external beam radiotherapy ( $2 \mathrm{~Gy}$ per fraction, five times a week, no alternative fractionation schemes like hyperfractionation were applied in our patient population). The first phase delivered a dose of 50 Gy to the primary tumor and associated nodal drainage sites. Subsequently, a boost was applied: In the primary setting, the boost included the primary tumor and macroscopic involved lymph node areas to a total dose of $70 \mathrm{~Gy}$. In the adjuvant situation, the boost was applied to the primary tumor region and tributary lymph node regions including such lymph nodes with extranodal spread to a total dose of $64 \mathrm{~Gy}$. Treatment was delivered with a Varian Clinac $600 \mathrm{C} / \mathrm{D}$ accelerator (Varian, Palo Alto, CA, USA). The prescribed 
dose was defined in accordance with the International Commission on Radiation Units and Measurement Report (International Commission on Radiation Units and Measurements Prescribing, recording and reporting photon beam therapy. ICRU report No. 50, Bethesda, MD: ICRU 1993). Simultaneous chemotherapy was given as follows to all patients in the study: Intravenous infusion of cisplatin $6 \mathrm{mg} / \mathrm{m}^{2}$ was combined with $11 \mathrm{NaCl}$ with facultative antiemetic medication on every radiotherapy day. Radiotherapy was applied after a minimum of $3 \mathrm{~h}$ following chemotherapy.

Toxicity was monitored weekly during radiochemotherapy and every second week following therapy until disappearance of acute toxicity. Subsequently, chronic toxicity was monitored at least quarterly. Toxicity was classified according to the CTC score for acute (Trotti et al. 2000) and according to the LENT scoring system for chronic toxicity (Rubin et al. 1995).

After radiochemotherapy, remission was evaluated by clinical ENT-examination (ear-nose-throat endoscopy, magnifying laryngoscopy, whenever necessary upper bronchoscopy, and esophagoscopy) and a computed tomography with contrast medium. Complete remission was defined as the complete regression of all tumor manifestations. Afterwards, patients underwent quarterly clinical ENTexamination, complete blood counts, biochemical analysis, chest X-rays, abdominal ultrasound or a computed tomography of the head and neck, if necessary. Biopsies were taken from suspect findings to receive histological confirmation of tumor growth.

Survival probabilities were calculated from the day of histological diagnosis. The Kaplan-Meier product-limit method was used to determine overall survival and locoregional control. Loco-regional control was defined as the absence of local or regional recurrence or progression (Kaplan and Meier 1958). The impact of possible prognostic factors was estimated by univariate analysis (log-rank test) for gender, patient age, primary site, tumor stage, histological grading, surgery, and preradiotherapeutic hemoglobin levels. Statistical analyses were generated with the program StatSoft, Inc. (2002) STATISTICA for windows version 6.1.

\section{Results}

Toxicity

In summary, therapy was well tolerated. There were no treatment-related deaths. Acute grade 3 sole mucositis/dysphagia was seen in 11 patients $(22 \%)$, sole hematologic toxicity $\geq$ grade 3 occurred in 7 patients (14\%) [5 leukopenia ( 3 of these patients with grade 4 toxicity), 1 patient thrombopenia grade 3 and one patient leukopenia and additionally anemia grade 3]. In another 4 patients $(8 \%)$ mucositis/dysphagia and hematologic toxicity grade 3 (2 leukopenia and 2 leukopenia and thrombopenia) was observed. Thus, toxicity related changes of the therapy schedule were infrequently necessary. Overall, $94 \%$ of our patients received the intended dose of radiotherapy (in 2 patients receiving adjuvant treatment radiotherapy dose was reduced from 64 to $60 \mathrm{~Gy}$ and from 64 to $62 \mathrm{~Gy}$, respectively and in one patient receiving primary radiochemotherapy from 70 to $62 \mathrm{~Gy}$ ). More than $80 \%$ of the intended cumulative chemotherapy dose could be applied in $90 \%$ of our patients (chemotherapy break for more than one week was necessary in 8 patients, only). During followup, high grade chronic toxicity (grade 3 ) was also infrequent and occurred in nine patients (18\%), only (3 patients xerostomia, 2 patients subcutaneous fibrosis, 2 patients lymphedema, and 2 patients subcutaneous fibrosis and lymphedema, respectively). No grade 4 chronic toxicity was observed in our patients during follow-up.

Follow-up, response and overall survival/loco-regional relapse, loco-regional control and development of distant metastases

All patients, who underwent surgery in curative intent, were at least macroscopically completely resected (28 patients R0-resection, 4 patients R1-resection). Complete remission after primary radiochemotherapy was seen in 12/ 18 patients $(66 \%)$. In the other six patients, a partial remission was achieved. The median duration of follow-up was 24.2 months (range, 7.8-48.7 months). After this time, 38 patients $(76 \%)$ remain alive. Death has occurred in 12 patients (24\%); 9 of these died from tumor, and 3 died from intercurrent disease (secondary primary tumor, pulmonary embolism and one not known). Collectively, the 2- and 3year overall survival rates were 72.7 and $67.1 \%$, respectively. Loco-regional relapse (recurrence after complete or progress after partial remission) occurred in ten patients (in 7 patients after adjuvant and 3 patients after primary radiochemotherapy); the median time to relapse was 9 months (range 0.8-13.9). In nine patients, the initial site of relapse was local and in one patient regional. Distant metastases occurred in two patients (one hepatic and one pulmonal), in both cases not associated with a loco-regional recurrence. Collectively, the 2- and 3-year loco-regional control rates were $78 \%$.

\section{Prognostic factors}

To evaluate the prognostic value of individual factors, univariate subgroup analyses concerning gender, patient age, primary site, tumor stage, histological grading, surgery, and 
preradiotherapeutic hemoglobin levels have been done. Subgroup analysis of tumor stage (stage III versus stage IVA/B) showed that disease stage III at the time of diagnosis is associated with a statistical significant $(P=0.02)$ higher 2- and 3-year year overall survival compared to the patients staged IVA/B (100 vs. $65.3 \%$, and 100 vs. $57 \%$, respectively). In contrast, subgroup analyses concerning gender, patient age, primary site, histological grading, surgery, and preradiotherapeutic hemoglobin levels showed no statistically significant influence on overall survival or loco-regional control. Still, this may be due to the small sample size.

\section{Discussion}

Toxicity of different chemotherapy regimens used in combined conventional fractionated primary or adjuvant radiochemotherapy for locally advanced HNSCC

The data show that our radiochemotherapy regimen using low dose cisplatin is associated with relatively low high grade ( $\geq$ grade 3 ) toxicity. Thus, $94 \%$ of the patients received the intended dose of radiotherapy and in $90 \%$ of the patients $\geq 80 \%$ of the intended cumulative chemotherapy dose could be applied. Other common chemotherapy regimens (cisplatin $100 \mathrm{mg} / \mathrm{m}^{2}$ on days 1,22 , and 43 of radiotherapy, $50 \mathrm{mg} / \mathrm{m}^{2}$ weekly during radiotherapy, or $20 \mathrm{mg} / \mathrm{m}^{2}$ for 4 days in week 1 and 5 of radiotherapy) are associated with equal or considerably more high grade acute toxicity: As shown in Table 2, the incidence of $\geq$ grade 3 mucositis/dysphagia/hematologic toxicity in these studies varies from 41 to $89 \%$. High acute toxicity may reduce quality of life in patients, and toxicity related changes of the therapy concept may be necessary which, however, may adversely influence prognosis. For example, Bernier et al. (2004) described 2004 that the third cycle of their chemotherapy (cisplatin $100 \mathrm{mg} / \mathrm{m}^{2}$ on days 1,22 , and 43 of radiotherapy) could be administered on time without delay in only $49 \%$ of their patients.

Our data are in accordance with other studies using daily low dose cisplatin in radiochemotherapy for locally advanced HNSCC: Jeremic et al. (2004) and Hoebers et al. (2007) also demonstrated that concurrent normofractionated chemoradiation with daily low dose cisplatin for advanced head and neck cancer patients is feasible and effective. Thereby, Jeremic et al. (2004) reported similar data concerning toxicity compared to our study, Hoebers et al. (2007), however, describe higher amounts (Table 2). Additionally, Zuur et al. (2008) considered this treatment scheme as a relatively safe protocol with respect to ototoxicity.
Our data show a 2-year/3-year overall survival rate of $72.7 \% / 67.1 \%$, and a 2-year/3-year loco-regional-control rate of $78 \%$, respectively. However, as both, patients with surgery and postoperative radiochemotherapy and patients with radiochemotherapy alone as definitive treatment are regarded, conclusions concerning oncological efficacy in comparison to the literature cannot be drawn from the data.

Efficacy and toxicity of hyperfractionated and/or accelerated radio- or radiochemotherapy regimens for primary treatment of inoperable locally advanced HNSCC

Whereas in the adjuvant situation conventional fractionated radiotherapy is the standard approach, overall survival and loco-regional control may be improved by alternative fractionation like acceleration or hyperfractionation when using radiotherapy as primary therapy for inoperable advanced HNSCC: It has been shown that hyperfractionation with moderate dose escalation leads to a significant improvement of loco-regional control and overall survival if radiation therapy is used as single modality. In contrast, accelerated radiation therapy alone, especially when given as split course radiation schedule, does not increase overall survival (Budach et al. 2006). However, no evidence proofs that hyperfractionation is better compared to conventional fractionation if chemotherapy is given concomitantly (Welz et al. 2007). Furthermore, it has to be considered that alternative fractionation is associated with higher acute toxicity and may lead to toxicity related changes of the therapy concept resulting in negative impact on prognosis. Potentially, daily low dose cisplatin is appropriate to reduce acute toxicity in hyperfractionated radiotherapy without compromising tumor control compared to regimens using higher single doses of cisplatin for radiosensitizing. In summary, despite hundreds of clinical trials in patients with advanced disease, there is no absolute consensus about patient selection for altered fraction regimens, type of chemo-radiotherapy association, radiation or chemotherapy dose schedule (Corvo 2007).

\section{Conclusion}

We found concurrent chemoradiation with daily low dose cisplatin to be feasible with advantage of low acute and chronic toxicity. Therefore, use of low dose cisplatin should be evaluated in future clinical trials testing conventional or alternative fractionated radiotherapy for locally advanced HNSCC. 


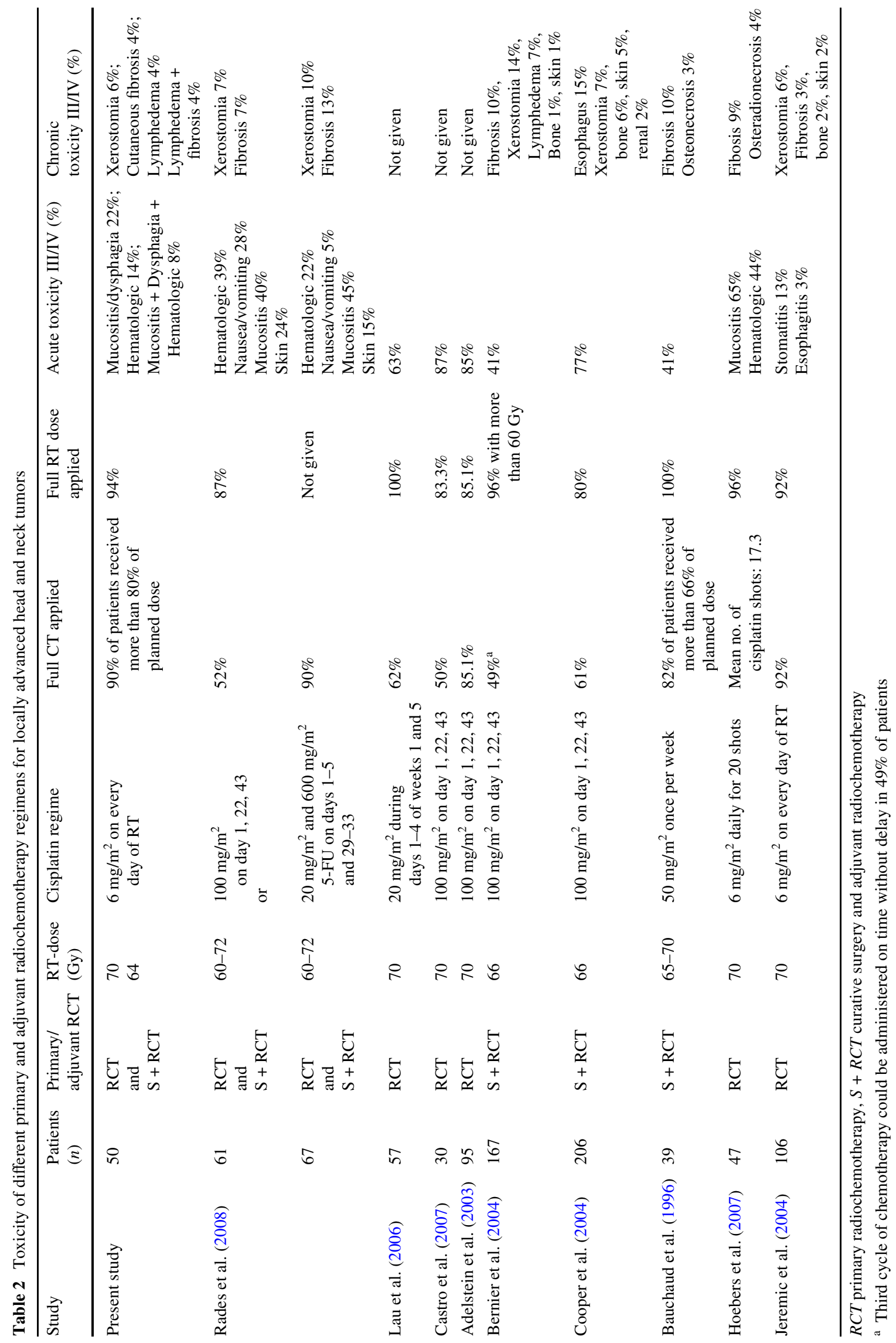


Conflict of interest statement We declare that we have no conflict of interest.

Open Access This article is distributed under the terms of the Creative Commons Attribution Noncommercial License which permits any noncommercial use, distribution, and reproduction in any medium, provided the original author(s) and source are credited.

\section{References}

Adelstein DJ, Li Y, Adams GL, Wagner H Jr, Kish JA, Ensley JF et al (2003) An intergroup phase III comparison of standard radiation therapy and two schedules of concurrent chemoradiotherapy in patients with unresectable squamous cell head and neck cancer. J Clin Oncol 21:92-98. doi:10.1200/JCO.2003.01.008

Adelstein DJ, Saxton JP, Rybicki LA, Esclamado RM, Wood BG, Strome M, Lavertu P et al (2006) Ma Multiagent concurrent chemoradiotherapy for locoregionally advanced squamous cell head and neck cancer: mature results from a single institution. J Clin Oncol 24:1064-1071. doi:10.1200/JCO.2005.01.5867

Bauchaud JM, Cohen-Jonathan E, Alzieu C, David JM, Serrano E, Daly-Schveitzer N et al (1996) Combined postoperative radiotherapy and weekly cisplatin infusion for locally advanced head and neck carcinoma: final report of a randomized trial. Int J Radiat Oncol Biol Phys 36(5):999-1004. doi:10.1016/S0360-3016(96) 00430-0

Bernier J, Domenge C, Ozsahin M, Matuszewska K, Lefebvre JL, Greiner RH et al (2004) Postoperative Irradiation with or without concomitant chemotherapy for locally advanced head and neck cancer. N Engl J Med 350:1945-1952. doi:10.1056/NEJMoa032641

Browman GP, Hodson DI, Mackenzie RJ, Bestic N, Zuraw L (2001) Choosing a concomitant chemotherapy and radiotherapy regimen for squamous cell head and neck cancer: a systematic review of the published literature with subgroup analysis. Head Neck 23:579-589. doi:10.1002/hed.1081

Budach W, Hehr T, Budach V, Belka C, Dietz K (2006) A meta-analysis of hyperfractionated and accelerated radiotherapy and combined chemotherapy and radiotherapy regimens in unresectable locally advanced squamous cell carcinom of the head and neck. BMC Cancer 6:28. doi:10.1186/1471-2407-6-28

Castro G, Snitcovsky IML, Gebrim EM, Leitao GM, Nadalin W, Ferraz AR et al (2007) High-dose cisplatin concurrent to conventionally delivered radiotherapy is associated with unacceptable toxicity in unresectable, non-metastatic stage IV head and neck squamous cell carcinoma. Eur Arch Otorhinolaryngol 264(12):1475-1482. doi:10.1007/s00405-007-0395-9

Cooper JS, Pajak TF, Forastiere AA, Jacobs J, Campbell BH, Saxman SB et al (2004) Postoperative concurrent radiotherapy and chemotherapy for high-risk squamos-cell carcinom of the head and neck. N Engl J Med 350(19):1937-1944. doi:10.1056/NEJMoa032646

Corvo R (2007) Evidence based radiation oncology in head and neck squamos cell carcinoma. Radiother Oncol 85(1):156-170. doi:10.1016/j.radonc.2007.04.002

El-Sayed S, Nelson N (1996) Adjuvant and adjunctive chemotherapy in the management of squamous cell carcinoma of the head and neck region A meta-analysis of prospective and randomized trials. J Clin Oncol 14:838-847

Geeta SN, Padmanabhan TK, Samuel J, Pavithran K, Lyer S, Kuriakose MA (2006) Comparison of acute toxicities of two chemotherapy schedules for head and neck cancers. JCRT 2(3):100-1004

Hoebers FJ, Heemsbergen W, Balm AJ, van Zanten M, Schornagel JH, Rasch CR (2007) Concurrent chemoradiation with daily low dose cisplatin for advanced stage head and neck carcinoma. Radiother Oncol 85(1):42-47. doi:10.1016/j.radonc.2007.03.014

International Commission on Radiation Units and Measurements Prescribing, Recording and Reporting Photon Beam Therapy (1993) ICRU report No. 50. ICRU, Bethesda

Jeremic B, Milicic B (2008) Influence of low-dose cisplatin on the distant metastasis-free survival of patients with locally advanced nonmetastatic head and neck cancer treated with radiation therapy. Radiother Oncol 87(2):201-203. doi:10.1016/j.radonc. 2007.12.023

Jeremic B, Milicic B, Dagovic A, Vaskovic Z, Tadic L (2004) Radiation therapy with or without concurrent low-dose daily chemotherapy in locally advanced, nonmetastatic squamous cell carcinoma of th head and neck. J Clin Oncol 22(17):3540-3548. doi:10.1200/JCO.2004.10.076

Kaplan E, Meier P (1958) Nonparametric estimation from incomplete observations. J Am Stat Assoc 58:457-481. doi:10.2307/2281868

Lau H, Brar S, Hao D, MacKinnon J, Yee D, Gluck S (2006) Concomitant low-dose cisplatin and three-dimensional conformal radiotherapy for locally advanced squamous cell carcinoma of the head and neck: analysis of survival and toxicity. Head Neck 28(3):189196. doi:10.1002/hed.20324

Pignon JP, Bourhis J, Domenge C, Designe L (2000) Chemotherapy added to locoregional treatment for head and neck squamous-cell carcinoma: three meta-analyses of updated individual data MACH-NC Collaborative Group. Meta-analysis of chemotherapy on head and neck cancer. Lancet 355:949-955

Pradier O, Lederer K, Hille A, Weiss E, Christiansen H, Schmidberger $\mathrm{H}$ et al (2005) Concurrent low-dose cispaltin and thoracic radiotherapy in patients with inoperable stage III non-small cell lung cancer: a phase II trial with special reference to the haemoglobin level as prognostic parameter. J Cancer Res Clin Oncol 131(4):261-269. doi:10.1007/s00432-004-0633-0

Rades D, Fehlauer F, Sheikh-Sarraf M, Kazic N, Basic H, Poorter R et al (2008) Toxicity of two cisplatin-based radiochemotherapy regimes for the treatment of patients with stage III/IV head and neck cancer. Head Neck 30(2):235-241. doi:10.1002/hed. 20683

Rubin P, Constine LSIII, Fajardo LF, Phillips TL, Wassermann TH (1995) EORTC Late Effects Working Group. Overview of late effects normal tissues (LENT) scoring system. Radiother Oncol 35:9-10. doi:10.1016/0167-8140(95)97447-L

Schaake-Koning C, van den Bogaert W, Dalesio O, Festen J, Hoogenhout J, van Houte P et al (1992) Effects of concomitant cisplatin and radiotherapy on inoperable non-small-cell lung cancer. $\mathrm{N}$ Engl J Med 326(8):524-530

Semrau S, Bier A, Thierbach U, Virchow C, Ketterer P, Klautke G et al (2007) 6-year experience of concurrent radiochemotherapy with vinorelbine plus a platinum compound in multimorbis or aged patients with inoperable non-small cell lung cancer. Strahlenther Onkol 183(1):30-35. doi:10.1007/s00066-007-1593-5

Takiguchi Y, Urama R, Asaka-Amano Y, Kuroso K, Kasahara Y, Tanabe $\mathrm{N}$ et al (2005) Phase I study of cisplatin and irinotecan combined with concurrent hyperfractionated accelerated thoracic radiotherapy for locally advanced non-small cell lung carcinoma. Int J Clin Oncol 10(6):418-424. doi:10.1007/ s10147-005-0525-6

Trotti A, Byhardt R, Stetz J, Gwede C, Com B, Fu K et al (2000) Common toxicity criteria: Version 2.0 an improved reference for grading the acute effects of cancer treatment: impact on radiotherapy. Int J Radiat Oncol Biol Phys 47:13-47. doi:10.1016/S03603016(99)00559-3

Welz H, Pöttgen C, Abu Jawad J et al (2007) Hyperfraktioniertakzelerierte (HAF) vs. konventionell fraktionierte (CF) Strahlentherapie und simultane Chemotherapie (CTx) mit Mitomycin C und 5-FU bei lokal fortgeschrittenen Kopf-Hals-Karzinomen: 
Monoinstitutionale Ergebnisse an 299 Patienten. Strahlenther Onkol 2007:94-95

Zuur CL, Simis YJ, Verkaik RS, Schornagel JH, Balm AJ, Dreschler WA, Rasch CR (2008) Hearing loss due to concurrent daily low-dose cispaltin chemoradiation for locally advanced head and neck cancer. Radiother Oncol 89(1):38-43. doi:10.1016/j.radonc. 2008.06 .003 